This item was submitted to Loughborough's Research Repository by the author.

Items in Figshare are protected by copyright, with all rights reserved, unless otherwise indicated.

\title{
The influence of engine demand map design on vehicle perceived performance
}

PLEASE CITE THE PUBLISHED VERSION

http://dx.doi.org/10.1504/IJVD.2001.005221

PUBLISHER

(c) Inderscience Publishers

VERSION

AM (Accepted Manuscript)

LICENCE

CC BY-NC-ND 4.0

REPOSITORY RECORD

Passmore, Martin A., A. Patel, and R. Lorentzen. 2010. "The Influence of Engine Demand Map Design on Vehicle Perceived Performance”. figshare. https://hdl.handle.net/2134/6646. 
This item was submitted to Loughborough's Institutional Repository (https://dspace.lboro.ac.uk/) by the author and is made available under the following Creative Commons Licence conditions.

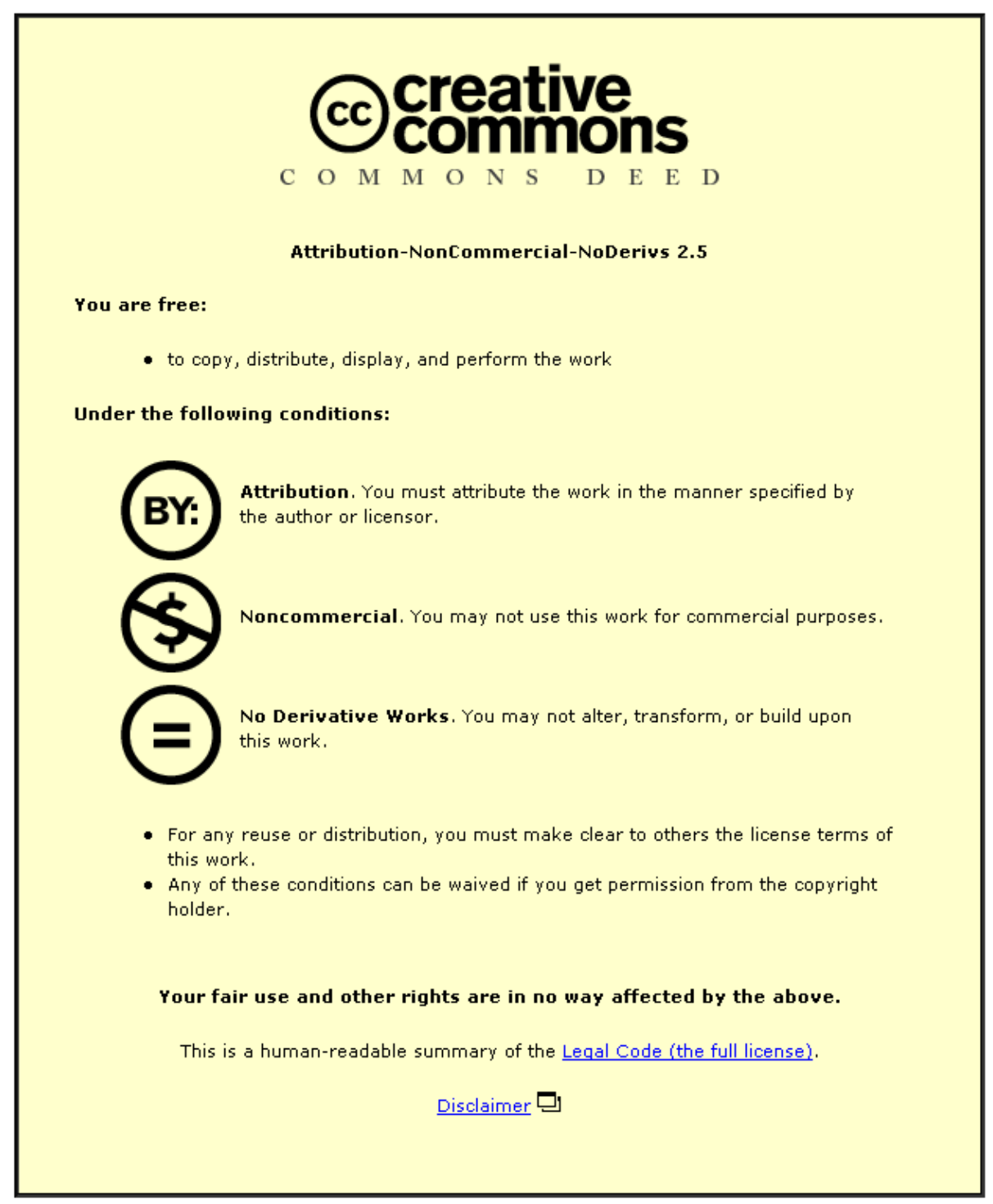

For the full text of this licence, please go to: http://creativecommons.org/licenses/by-nc-nd/2.5/ 


\title{
The influence of engine demand map design on vehicle perceived performance.
}

\author{
(c) Dr Martin A Passmore \\ Loughborough University \\ Loughborough \\ Leics, LE11 3TU
}

\author{
Dr Ash Patel, Mr Rob Lorentzen \\ Ford Motor Company
}

Published in International Journal of vehicle design. Vol 26, No5, 200, pp 509-522

\begin{abstract}
This paper reports on a study into the influence of the steady state engine demand map on perceived performance. An analysis of the results of a survey of twenty-four $\mathrm{C}$ and $\mathrm{C} / \mathrm{D}$ class $1600 \mathrm{cc}$ cars is presented and shows that the primary parameters are the mean wide-open throttle acceleration, the throttle progression, and the part throttle rate of change of acceleration with engine speed.

These results are used to design a factorial experiment to investigate these parameters using an electronic throttle system. This approach eliminates problems of inter-vehicle variations in noise, comfort or general image, allowing the subjective ratings to be attributed directly to the demand map changes. The results are discussed in terms of the significant main effects and interactions and as response surfaces, from which optimum set-ups can be determined.
\end{abstract}

\section{Keywords}

Perceived performance, demand map, drive-by-wire, subjective performance.

\section{Introduction}

The demands of the driving public, intense competition within the automotive industry and the progressive tightening of regulations in a number of areas has ensured that car manufacturers are continually striving to improve the vehicles they produce. Over the last decade significant improvements have been made in virtually all areas, for example, reliability, $\mathrm{NVH}$, emissions etc. and across all classes of car. One area that has come in for particular attention is the straight-line performance.

Vehicle straight-line performance has historically interested both the public and the motoring press. Figures such as maximum engine power, maximum vehicle speed and the time from 0 to $60 \mathrm{mph}$ are regularly quoted and compared. Where they have seen some apparent advantage motor manufacturers too have been happy to utilise such data in advertising. In practice, designers and engineers have understood for some time that such performance measures are not adequate indicators of the performance generally experienced on the road. This inadequacy arises because the measures are largely derived from the wide open throttle response; although this may to some degree indicate the vehicle's potential, it is not representative of the majority of real driving. Passmore(1990). If designers and engineers are to ensure that a new vehicle will be regarded as having good performance in practical use, then much better predictors of the perceived performance are required.

When assessing vehicle performance in a subjective sense the driver is exposed to a combination of quasi steady state performance, such as the acceleration through a speed range, dynamic effects, which may be characterised as vehicle response, and actual drive-line dynamics. In addition the assessment may be influenced by preconceptions concerning the vehicle and additional factors such as $\mathrm{NVH}$, handling and comfort.

This paper reports on a study into methods of improving the perceived performance through modifications to the steady state demand map. The steady state demand 
map is similar to the conventional engine map of torque against speed for constant throttle angle, but is defined as either torque or acceleration against speed for constant throttle pedal position. Hence the use of the term demand map.

The pilot study considers the relationship between subjective ratings of a selection of similar class current vehicles and a broad range of measured and derived demand map parameters. The results from this are used to design a main experiment to study the important factors using a more sophisticated approach employing a drive-by-wire throttle system. Before discussing these two studies and the results obtained a brief summary of the subjective assessment methodology is presented.

\section{Subjective assessment methodology}

Drive appraisal and route design: The preferred method for the drive appraisal in the work described here is an accompanied drive around a prescribed test route. This method is efficient in test time, relatively straightforward to perform, and can be designed to avoid bias. Although a route which encompasses the national mix of road types can be designed, and may be useful for fuel economy studies; previous subjective performance work, Passmore (1990), has shown that the most consistent ratings are obtained from drives performed on $A$ and $B$ roads. As the subjects are not requested to perform any specific tests or manoeuvres the appraisal may be referred to as a free driving format.

Response variables: The subjective test method ultimately relies on the subject providing quality feedback on the vehicle. The response variables must be chosen to best achieve this. Having adopted a free driving format for the appraisal, response variables that can be interpreted by the subjects in a relatively loose fashion are generally considered to be the most reliable.

A total of six response variables are used. (The names in brackets are used as abbreviations in some later plots)

\section{Overall performance \\ Responsive to accelerator depression \\ Smooth acceleration \\ Quick off the mark \\ Good acceleration through the gears \\ Ease of control of available power}

(RESP)

(SMOOTH)

(QUICK)

(GEARS)

(CONTROL)

Rating system: An important aspect of the test methodology is the choice of rating system. In general the subjects response is recorded using a simple scale, with the choice of scale depending on the particular rating task. It is also well understood, Osgood (1967) that subjective ratings are by their nature always comparative and that where possible the reference condition must be controlled. In the tests reported here all ratings were given relative to a standard vehicle.

Essentially the choice lies between a Likert type scale and a Semantic Differential scale. In general the Likert scale is preferred when the attribute being measured is complex and the Semantic Differential when the attribute covers a single aspect. To ensure the highest possible quality data a short experiment was conducted to establish the preferred scale for the drive appraisals. Passmore (1996). The results were analysed in terms of driver to driver variability in rating and the ability to resolve large and small changes in vehicle configuration. In all cases the subjects were provided with tuition at the start of the appraisal. Osgood (1967).

The performance of the scales is assessed by comparing the variation as a percentage of the total scale (i.e. the full-scale deflection), and the movement along the scale as a percentage of full scale. The differences in results between the scale types were relatively small with the Semantic Differential scale yielding lower 
variability for some response variables and test configurations, and the Likert scale proving better for others. However the magnitude of change in response was higher using the Likert scale, indicating that it is better for recording small changes. Figure 1 shows an example Likert scale as used throughout the appraisals. Based on twelve subject repeats the overall accuracy of the mean rating across all response variables is approximately \pm 0.5 of a rating. The accuracy for the overall performance response variable is significantly better at approximately \pm 0.25 of a rating.

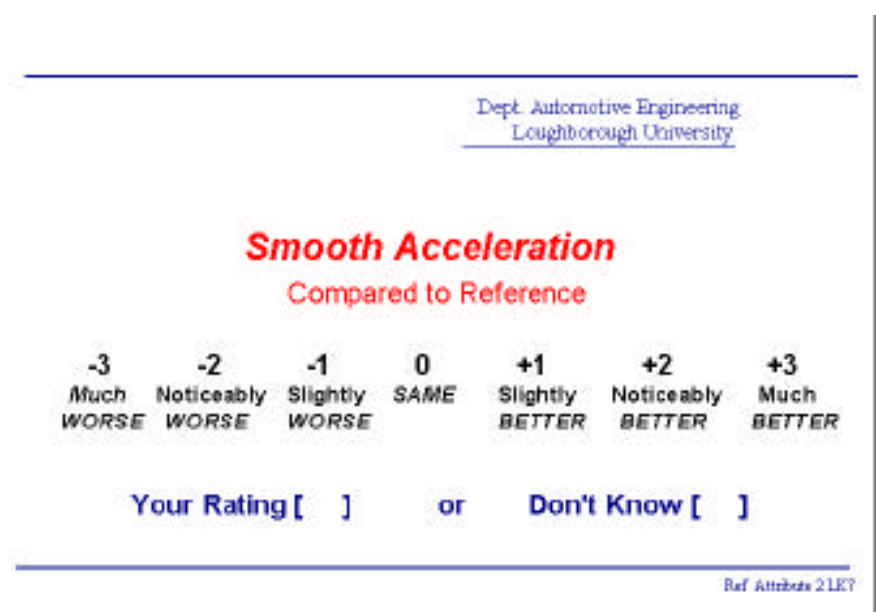

Figure 1 Likert rating scale.

\section{Pilot study.}

Three expert drivers rated twenty-four similar size 1600cc cars that were subsequently tested on a chassis dynamometer to measure their demand maps. Expert in this context simply implies that they are experienced in rating vehicles and have been shown to produce results close to the average for a group of members of the public. Using the dynamometer data as input to a performance simulation program a range of performance parameters were calculated. These were tested against the subjective ratings using non-parametric statistics. (Kendal's rank correlation $\tau$ ). The example shown in Figure 2 shows the correlation between the ranking obtained from the subjective ratings and the ranking of time to accelerate from 50 to $100 \mathrm{kph}$ in top gear (shortest time ranked $1^{\text {st }}$ and longest time ranked $24^{\text {th }}$.) The Kendal rank correlation coefficient $(\tau)$ is 0.69 with a $p$ value of $0.24 \times 10^{-5}$. This latter value being the probability that the value of $\tau$ could have arisen by chance.

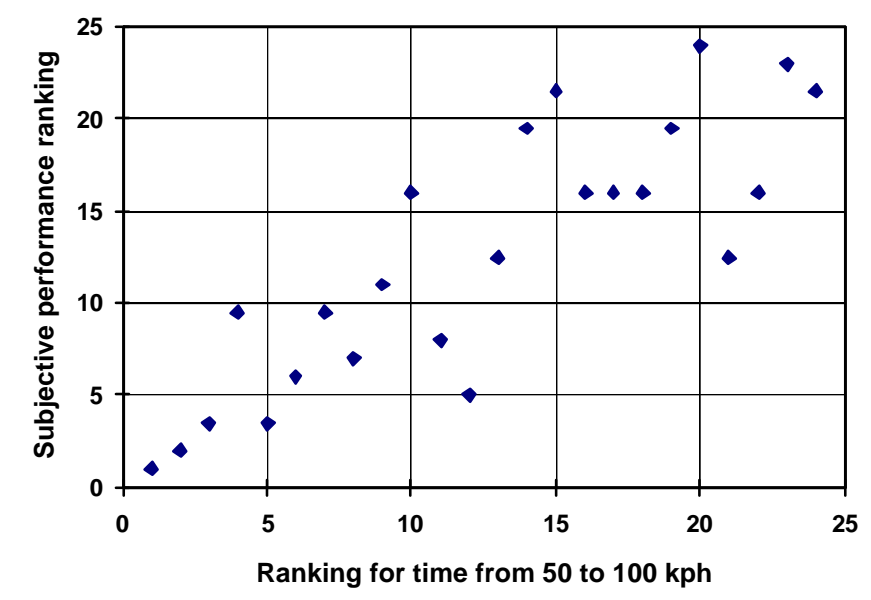

Figure 2. Example competitor survey correlation. 
Several other parameters also gave rise to correlation's with coefficients of the order of 0.69 for example:

- Mean acceleration at wide open throttle between idle and $5000 \mathrm{rpm}$

- Through the gears 0-100 kph time, with gear change speeds determined from customer data

- Ratio of torque at wheels to mass

These parameters are however, all measures of similar attributes derived from the wide open throttle characteristic. It is concluded that any one of them would provide an equally good prediction of the subjective performance but they cannot be combined to improve the prediction because they are highly correlated with each other. They also illustrate that some of the traditional parameters used to indicate performance do have some sound basis for use. It is noted however that the correlation for parameters such as maximum power, maximum torque, and the zero to $100 \mathrm{kph}$ time using gear change speeds optimised for minimum time are significantly lower (Kendal $\tau \approx 0.49$ ).

Some additional parameters less correlated with those in the first group also showed significant results yielding coefficients of the order of $\tau=0.55$. Two examples are:

- Throttle progression

- Mean acceleration at part throttle.

Parameters within this group are also measures of a similar attribute though in this case derived from part throttle rather than the wide open throttle characteristic. The overall worth of the data can be improved using multiple regression to summarise the relationship between all of the correlating factors and the subjective performance. In the process it will identify a subset of independent variables which are most useful in predicting performance feel, while rejecting all but the best predictor in any group of correlated variables.

The regression used a stepwise selection process with a total of twenty-five parameters shown to correlate with subjective performance. The entry and removal criterion used was a probability of 0.05 that the coefficient is not zero for entry, and 0.1 for removal. The final model included only two variables, the mean wide-open throttle acceleration in 5th gear, which accounted for $63 \%$ of the variance, and the throttle progression which accounted for an additional 10\%. Relaxing the entry criterion to 0.1 then the part throttle slope $(\mathrm{da} / \mathrm{dN})$ is also entered into the model explaining a further $3 \%$ of the variance.

The relative importance of the parameters in any study of this sort is determined to some extent by the range of values for each parameter being investigated. This has been controlled to some extent by confining the analysis to a single engine capacity. However the relatively small influence of the progression and da/dD may only indicate that most of the vehicles considered are reasonably close to the optimum for progression, and all have very similar values of da/dN. The latter being the natural characteristic of a SI engine fitted with a butterfly throttle valve, where, at part throttle the available torque reduces slowly with increasing engine speed.

The pilot study shows that the most important determining factor in perceived performance is the wide-open-throttle characteristic, i.e. the actual potential for acceleration. However the nature of the experiment makes it difficult to clearly quantify the influence of the secondary parameters. Particularly as they may be partially confounded by uncontrolled effects such as the individual vehicles image, comfort or driveability. A technique allowing control of these secondary factors was considered necessary to further the study. This gave rise to the development of a drive-by-wire throttle system. 


\section{Drive-by-wire throttle system.}

A fully mapped drive-by-wire throttle system essentially interprets the driver demand (i.e. throttle pedal position) and engine speed using some pre-determined strategy to drive a position control actuator connected to the throttle plate. The advantage of the system is that a number of different performance characteristics can be implemented on a single vehicle. Distortions caused by inter vehicle variations in $\mathrm{NVH}$, ride and handling, comfort or even overall image of the vehicle are thereby removed, and recorded ratings can be directly attributed to the change that has been made. A more detailed description of a system similar to that described here is available in the literature. Emtage (1991).

The drive-by-wire calibration defines throttle actuation in terms of driver demand (pedal position) and engine speed and is formed by combining a driver demand map and a standard engine torque map. Essentially the demand map specifies the torque or acceleration as a function of throttle pedal position and engine speed. The engine map specifies the torque as a function of throttle plate angle and engine speed. In this work the demand maps are specified in terms of the acceleration in first gear.

The demand map is designed by first specifying the required maximum acceleration across the engine speed range. This must clearly not exceed the actual capabilities of the engine. For reasons of safety the zero demand is always taken as the overrun curve of the standard vehicle to provide the expected degree of engine braking. The internal characteristics can be specified by any number of strategies but in this case two simplifying parameters, the progression, and the part throttle slope are used to specify the complete internal characteristic. The progression is the rate of change of acceleration with driver demand $(\mathrm{da} / \mathrm{dD})$ and is specified in terms of acceleration per percent pedal travel $\left(\mathrm{ms}^{-2} / \%\right)$. The part throttle slope $\left(\mathrm{da} / \mathrm{dN} \mathrm{NT}_{\mathrm{PT}}\right)$ is specified in terms of acceleration per rpm $\left(\mathrm{ms}^{-2} / \mathrm{rpm}\right)$. In both cases this is the first gear acceleration. Figure 3 shows an example demand map with a progression of $0.05 \mathrm{~m}^{-2} / \%$, and a part throttle slope of $-0.0002 \mathrm{~ms}^{-2} / \mathrm{rpm}$.

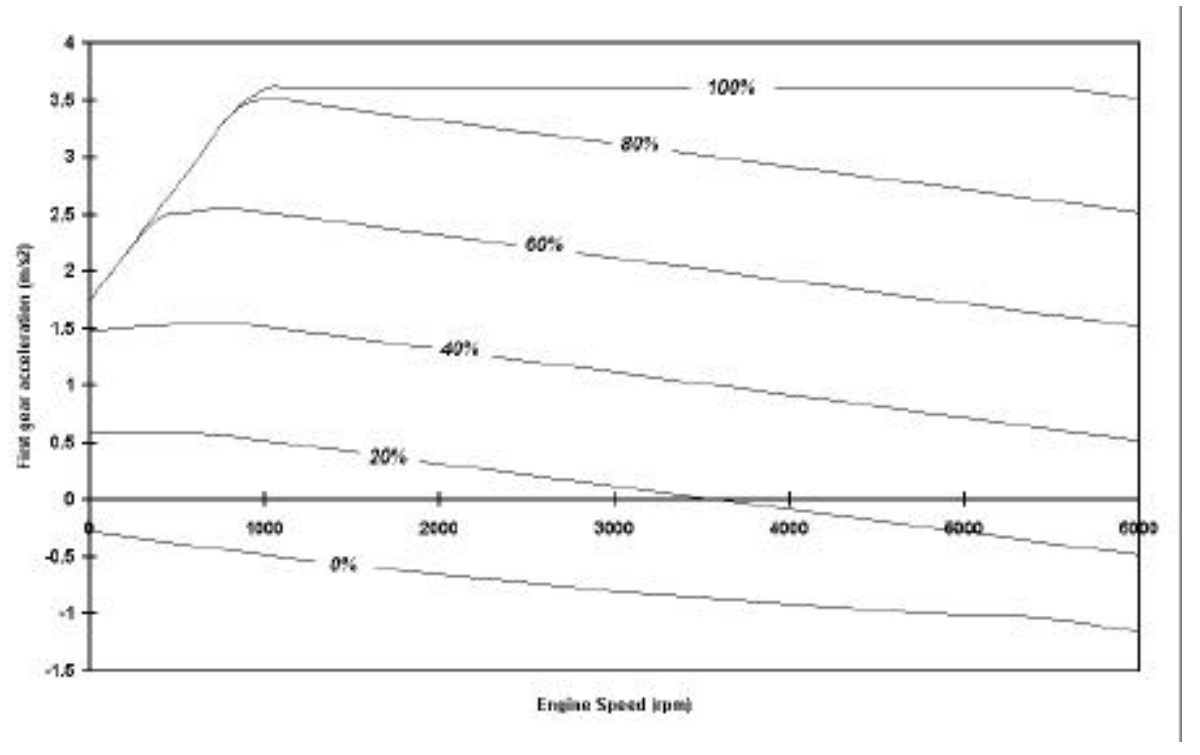

Figure 3. Demand map

The chosen strategy for defining the map ensures that the progression is constant (almost) throughout the speed and demand range, up to the point of saturation. Further pedal travel then yields no further response from the vehicle. This is quite different to a real vehicle where the progression tends to reduce towards maximum acceleration in a characteristic $S$ shape. This can be seen in Figure 4 where the acceleration is plotted against demand for a fixed engine speed. 


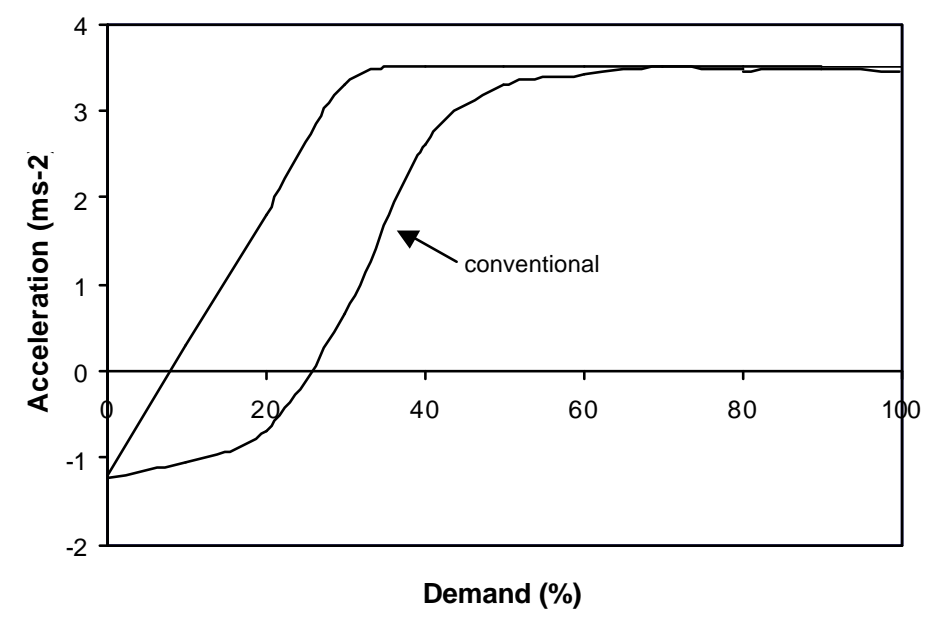

Figure 4 Comparison of conventional and drive-by-wire progression curves

The final drive by wire calibration map is determined by combining the required demand map with the host vehicle's inherent characteristic to determine the required throttle opening for any given driver demand and engine speed. This can be performed in advance to generate a two-dimensional look up table. The electronic throttle system uses this data when the vehicle is in use to generate the required actuator position. To improve resolution the software interpolates between points in the calibration file.

In the form used in the work reported here the system was installed on a V6 C/D class car and was configured to make seven different calibrations available to test observer via a simple handset. The use of a V6 provided the required map envelope to study the parameters at appropriate levels.

\section{Factorial study.}

The parameters identified in the pilot study can be summarised as; the level of the wide open throttle acceleration curve, the throttle progression and the part throttle acceleration curve slope. In addition the high correlation for 50 to $100 \mathrm{kph}$ time suggest that good acceleration at low engine speeds is an advantage, prompting the inclusion of a further parameter; the wide-open throttle slope.

The most straightforward experimental design would be to include the four parameters in a factorial experiment allowing all main effects and interactions to be determined. However the wide-open-throttle level and slope parameters cannot be altered independently, so the wide open throttle slope is removed from the main experiment reducing it to a three factor three level factorial design. The effect of the wide-open-throttle slope is the subject of a separate study.

A full factorial design is used to allow all main effects and interactions, if they exist, to be determined. All three parameters are tested at three levels, as there is some evidence in previous work of the presence of quadratic effects. Passmore (1994) The levels were set based on the results of previous studies, Passmore (1994) and in the case of wide-open throttle level by using the best and worst in class figures.

\begin{tabular}{|l|l|l|l|}
\hline & WOT level $\left(\mathbf{m s}^{-2}\right)$ & Progression $\left(\mathbf{m s}^{-2} / \%\right)$ & Part throttle slope $\left(\mathbf{m s}^{-2} / \mathbf{r p m}\right)$ \\
\hline high & 4.0 & 0.2 & 0.0002 \\
\hline mid & 3.6 & 0.125 & 0.0 \\
\hline low & 3.2 & 0.05 & -0.0002 \\
\hline
\end{tabular}

Figure 5 Factor settings. 
Figure 6 shows the overall design of the experiment, with a total of 27 test cells. The design is fully balanced ensuring that when the effect of one factor is calculated the effects due to other factors cancel out.

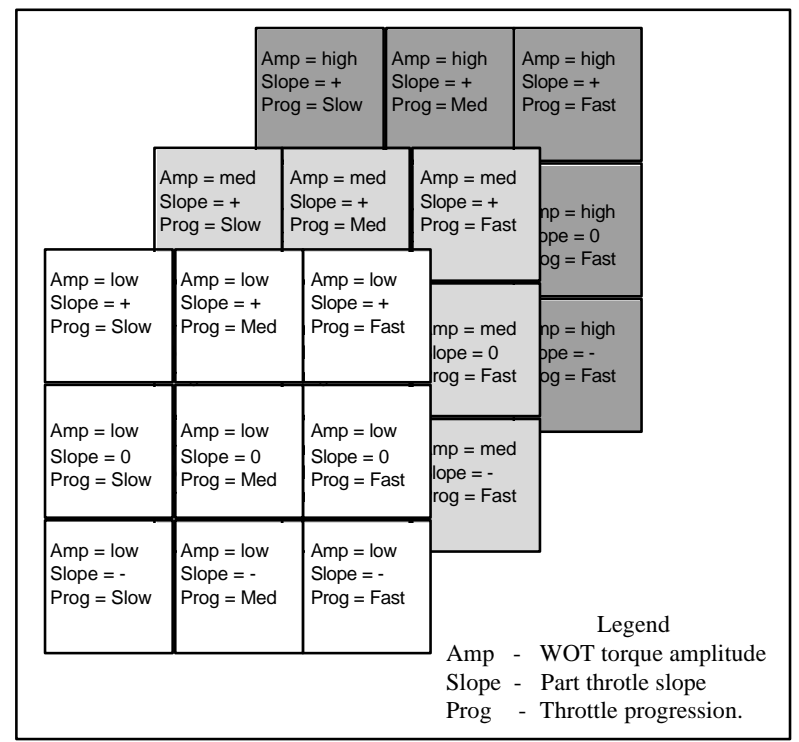

Figure 6. Main experiment design

A total of 16 repeat tests were performed in each cell, and the order of testing was fully randomised. Subjects were selected from the general public, confined to those who normally use or choose a vehicle in the test vehicle class. The central cell, where all three parameters are at the middle setting, was used as the reference vehicle.

\section{Analysis of the primary response variable. (SP)}

Compiling a rank order for the 27 test configurations based on the mean overall performance rating (SP) can reveal a first indication of the effect of the experimental variables.

The most striking result is that the nine configurations with the slowest throttle progression setting constitute the nine worst rankings. The influence of the two remaining parameters can be seen if we consider the arrangement of the top 18 configurations. It is clear that the high values of wide open throttle acceleration are biased towards the top of the group and the low values towards the bottom, leading to the conclusion that there is an effect due to wide open throttle level. It is also noted that the highest ranked configuration with wide open throttle torque at the middle setting has the most rapid progression, and a positive part throttle slope. This further reinforces the proposition that the progression is important, and may be evidence that there is an effect due to part throttle slope. 


\begin{tabular}{|c|c|c|}
\hline \begin{tabular}{|c|} 
wide open throttle \\
level
\end{tabular} & Progression & PT slope \\
\hline$(\mathrm{m} / \mathrm{s} 2)$ & (m/s2/\%) & (m/s2/rpm) \\
\hline high & high & mid \\
\hline high & mid & mid \\
\hline mid & high & high \\
\hline high & high & low \\
\hline high & mid & high \\
\hline high & high & high \\
\hline mid & high & Iow \\
\hline mid & mid & low \\
\hline high & mid & Iow \\
\hline Iow & high & mid \\
\hline Iow & high & Iow \\
\hline mid & mid & high \\
\hline mid & high & mid \\
\hline low & mid & low \\
\hline mid & mid & mid \\
\hline low & mid & mid \\
\hline Iow & high & high \\
\hline Iow & mid & high \\
\hline mid & Iow & mid \\
\hline high & Iow & high \\
\hline mid & Iow & high \\
\hline low & Iow & mid \\
\hline Iow & Iow & low \\
\hline Iow & low & high \\
\hline high & Iow & low \\
\hline mid & Iow & low \\
\hline high & Iow & mid \\
\hline
\end{tabular}

Figure 7 Ranking of configurations.

At this stage it is clearly necessary to embark on a formal analysis of the data through the calculation of the main effects (linear and quadratic), interactions and statistical significance. Three main effects are significant at the 0.05 level; the linear effect due to progression, followed by the quadratic effect due to progression and the linear effect due to changes in wide open throttle level. The linear interaction between wideopen throttle level and progression is also significant. Pooling those effects that are not significant into the error term the ANOVA table is shown in Figure 8.

\begin{tabular}{|l|l|l|l|l|l|}
\hline Factor & SS & dof & MS & F & D \\
\hline WOT Level (Linear) & 24.09 & 1 & 24.09 & 9.67 & .002003 \\
\hline Progression (Linear) & 199.50 & 1 & 199.50 & 80.12 & .000000 \\
\hline Progression (Quadratic)) & 40.96 & 1 & 40.96 & 16.45 & .000060 \\
\hline $\begin{array}{l}\text { Interaction between WOT Level } \\
\text { and progression }\end{array}$ & 11.55 & 1 & 11.55 & 4.64 & .031837 \\
\hline Lack of Fit & 51.65 & 22 & 2.347 & .942 & .537853 \\
\hline Pure Error & 971.03 & 390 & 2.490 & & \\
\hline Total SS & 1298.7 & 416 & & & \\
\hline
\end{tabular}

Figure 8 Table of ANOVA

The mean square errors (MS) show the relative contributions to the overall variation, with values of the $F$ test and the significance level $(p)$. Replication within the experiment (i.e. a total of 16 repeats per cell) allows the pure error associated with the measurements to be determined by calculating the variability within a set of repeats. $A$ lack of fit estimate is then also available, and is a test of residual variance in this case it is not significant $(p=0.526)$ indicating there are no unaccounted for effects. 
The relative magnitude of the significant effects is not important as these are partially due to the choice of levels tested. The important consideration is the ability to construct a response surface using these significant effects in order to set optimums and determine the sensitivity of the parameters.

The response surface for overall performance is shown in Figure 9. It includes only those variables that are significant at the 0.05 level.

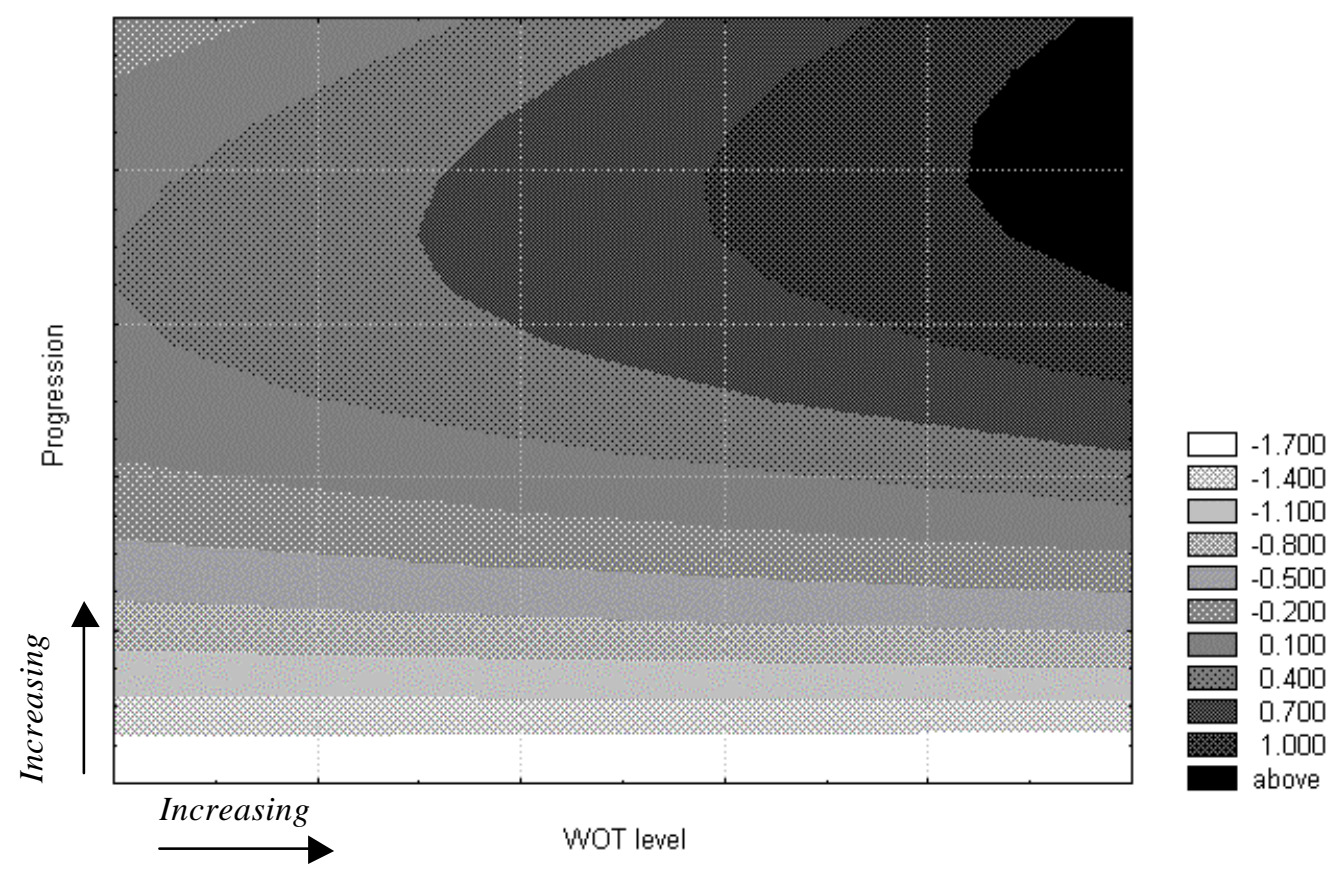

Figure 9 Response surface - Overall performance

As the effect due to da/dN is not significant this response surface represents the entire important parameter space. Contour values are the measured subjective response. The effects due to progression, wide-open throttle level and the interaction can all be identified in this response surface.

Changing parameters such as the progression alter the ratings for several reasons. It is clear from the competitor vehicle studies that acceleration is the primary influence and by changing the progression the actual acceleration experienced for a given pedal position is changed. In addition though it alters the driver's perception by changing the sensitivity (gain) of the throttle pedal. This changes both the response of the vehicle and its control. The interaction shown to be significant is that between the linear effect of wide open throttle level and the linear effect of progression. This indicates that if the wide open throttle acceleration is reduced then the value of progression required to give the optimum overall performance rating is also lower.

The presence of this interaction may be explained by a requirement for a minimum total active pedal travel. Two vehicles with the same throttle progression and part throttle slope but different levels of wide open throttle acceleration are identical up to the point of saturation at the lower level, and therefore exhibit exactly identical pedal sensitivities and acceleration characteristics. Further demand then produces no additional response in one vehicle, and continued response from the other. The reduction in progression implied by this interaction may be required to restore the total amount of active pedal travel to some acceptable level, albeit with some reduction in sensitivity. In the case of the response surface generated here this explanation is reinforced when it is calculated that the inclusion of the interaction reduces the 
variation in active pedal travel to $1 \%$ across range tested. This compares to $7 \%$ if the interaction is excluded.

The derivatives of the equation representing the surface in Figure 9 can be used as generic predictors. The fitted surface yields a function of the form:

$$
\text { Rating }(z)=a_{1}-a_{2} y+a_{3} x-a_{4}(x)^{2}+a_{5} y x
$$

where $y=$ WOT accelerati on. and $x=$ progressio $\mathrm{n}$

The partial derivatives with respect to wide-open throttle acceleration and progression are:

$$
\text { and } \begin{aligned}
\frac{\partial z}{\partial x} & =a_{3}-2 a_{4} x+a_{5} y \\
\frac{\partial \mathrm{z}}{\mathrm{y}} & =-a_{2}+a_{5} x
\end{aligned}
$$

At the optimum point both partial derivatives are zero and can be equated to yield the best value of progression for any value of wide-open-throttle acceleration.

$$
x=\frac{a_{5} y+\left(a_{2}+a_{3}\right)}{\left(2 a_{4}+a_{5}\right)}
$$

The presence of an interaction between wide-open-throttle acceleration and the progression has a number of implications. A simple application of the result is that if the wide open throttle acceleration curve is not flat, as it is in the case of this experiment, then the optimum progression required will be different at different engine speeds.

\section{Analysis of additional response variables.}

So far only the primary response variable, the overall performance (SP) has been considered. The following table illustrates the relationship between the ratings for all of the response variables. The correlation coefficients are Kendal's $\tau$.

\begin{tabular}{|l|l|l|l|l|l|l|}
\hline Variable & RESP & SMOOTH & OUICK & CONTROL & GEARS & SP \\
\hline RESP & 1.000 & 0.370 & 0.615 & 0.312 & 0.607 & 0.701 \\
\hline SMOOTH & 0.370 & 1.000 & 0.198 & 0.537 & 0.282 & 0.435 \\
\hline QUICK & 0.615 & 0.198 & 1.000 & 0.151 & 0.604 & 0.589 \\
\hline CONTRO1 & 0.312 & 0.537 & 0.151 & 1.000 & 0.255 & 0.403 \\
\hline GEARS & 0.607 & 0.282 & 0.604 & 0.255 & 1.000 & 0.667 \\
\hline SP & 0.701 & 0.435 & 0.589 & 0.403 & 0.667 & 1.000 \\
\hline
\end{tabular}

Figure 10 Correlation across response variables

The response variables can be divided into two groups; the first contains SP, RESP, QUICK and GEARS, and the second CONTROL and SMOOTH. Within these groups the correlations are relatively high, but across groups they are significantly lower. Analysing any of the parameters in the first group produces similar results to those discussed for SP. The results for CONTROL and SMOOTH however show some differences.

These two response variables were designed to measure the overall controllability of the vehicle during acceleration. At the 0.05 significance level the quadratic effect of progression and wide-open throttle level are significant. The response surface generated is shown in Figure 11. The axes cover the same range as that in Figure 9. It can be seen that there is a single optimum value of progression and wide open throttle level. Though in the case of the latter it is a very small effect. The optimum progression is significantly lower than that for maximum overall performance suggesting the need for some trade off in this area. 


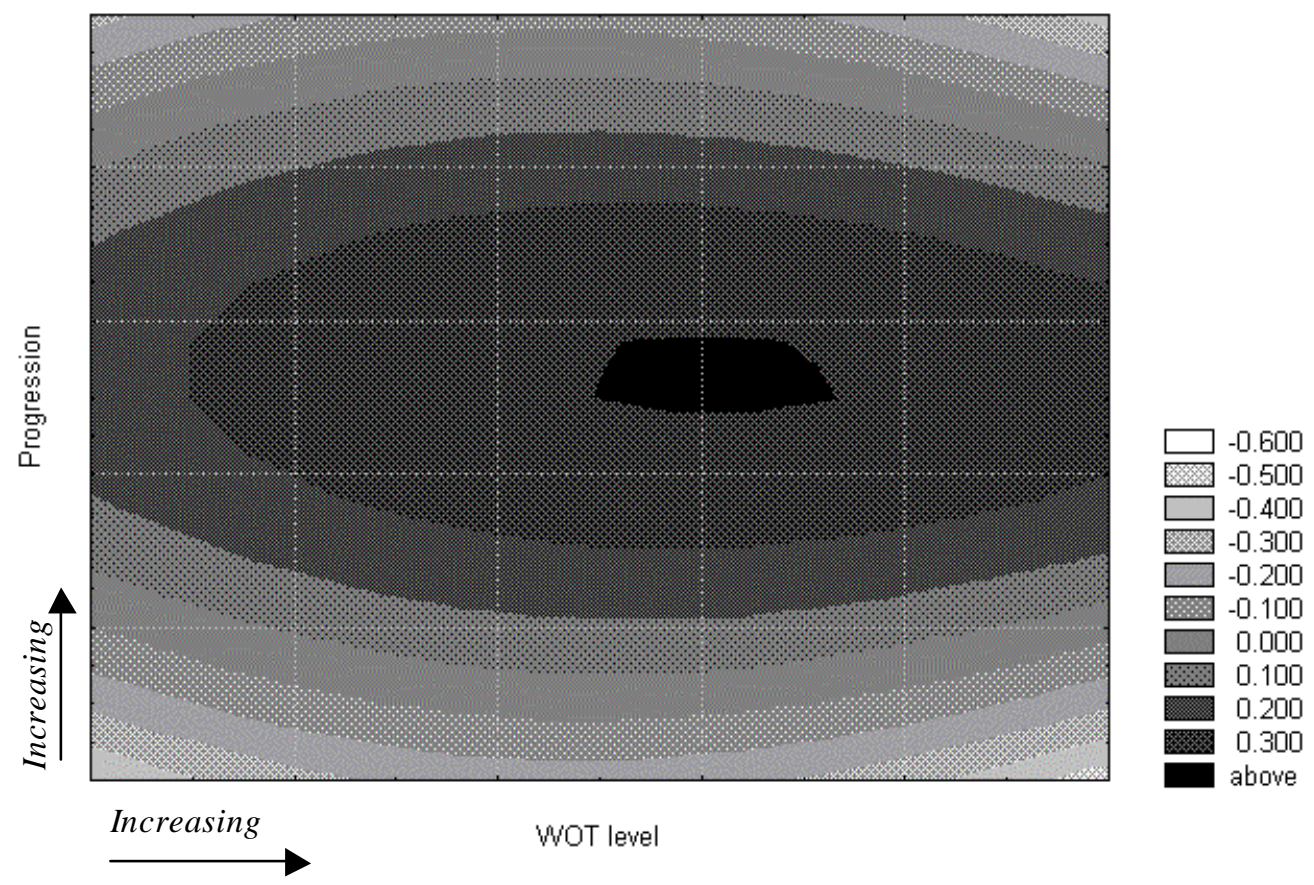

Figure 11 Response surface - Ease of control.

The issue of control within a system that comprises the vehicle and the driver is a complex one that requires an understanding of the driver's cognitive processes, motor control, the vehicle characteristics and the feedback paths to the driver.

At a rudimentary level the throttle pedal is the drivers main control device and its operation can be interpreted for both rapid and slow progressions. In the case of too low a progression as the driver steps in, let us assume to pull away, the car does not respond sufficiently to stimulate levels of acceleration (or other parameters) that are above the drivers' perception threshold. In the absence of this feedback the driver has no means of knowing how much more to step in, and experiences poor control. If the progression is too rapid the feedback is present but the drivers' motor control is not good enough to avoid overshoots, again this is interpreted as poor control.

\section{Conclusions}

- A study of 24 similar class vehicles showed that the potential acceleration of the vehicle (i.e. wide-open throttle acceleration) is the primary parameter in determining the perceived performance. Throttle progression and part-throttle slope were shown to be important secondary parameters.

- Multiple regression of the data from the 24 cars showed that the mean wide-open throttle acceleration in 5th gear, accounted for $63 \%$ of the variance, and the throttle progression accounted for an additional $10 \%$. Relaxing the entry criterion from 0.05 to 0.1 then the part throttle slope $(\mathrm{da} / \mathrm{dN})$ is also entered into the model explaining a further $3 \%$ of the variance.

- The best traditional performance parameters for indicating overall perceived performance are those such as fixed gear $50-100 \mathrm{kph}$ time and torque to weight ratio.

- An electronic throttle system was employed to allow control of the engine map design parameters whilst eliminating all other factors. The system was used in a factorial experiment to investigate effects of wide-open throttle acceleration, throttle progression and part-throttle slope. 
- In the assessment of overall perceived performance from the main factorial experiment the following effects were found to be statistically significant at the 0.05 level: linear progression, quadratic progression, linear effect of wide-open throttle acceleration level, and the interaction between wide-open throttle acceleration level and progression. Part throttle slope was not significant.

- A response surface for perceived performance showing the trade off between the significant variables has been generated.

- The presence of an interaction indicates that the optimum progression for bestperceived performance is a function of the level of wide-open throttle acceleration. It is suggested that this is required to maintain a set level of active throttle pedal travel.

- Analysis of the ratings for a number of response variables shows that they can be separated into two groups: in the first group, responsive to accelerator depression, quick off the mark, acceleration through the gears and overall performance feel, and in the second, smooth acceleration and ease of control. Within these groups the ratings are highly correlated.

- Analysis of the results for smooth acceleration and ease of control suggest that there is a single optimum value of progression which is lower than that required for optimum perceived performance.

\section{Acknowledgements}

The authors would like to thank the Ford Motor Company for supporting this work, and for giving permission to publish this paper.

Thanks are due to Jon Wheals for his work on the drive by wire system and the factorial study and to Dan Hatcher, Michael Huthnance and Dan Lintell for their tireless work recruiting subjects and running the appraisals.

\section{References}

Emtage AL, et al, The Development of an Automotive Drive-by-wire Throttle System as a research tool. Paper No. 910081, International Congress and Exposition, Detroit, Michigan, March 1991.

Osgood CE, Suci GJ, Tannenbaum PH, The measurement of meaning. University of Illinois press, 1967

Passmore MA, Lucas GG, Engineering aspects of performance feel, Volume 1, No. TT90R02, Department of Aeronautical and Automotive Engineering, Loughborough 1990.

Passmore MA, Wheals J, Model for Drivers' Perception of Vehicle Performance. . Paper No. 940386, International Congress and Exposition, Detroit, Michigan, Feb 1994.

Passmore MA, A factorial Study of Performance Feel: Factors effects and interactions. No. TT95R06, Department of Aeronautical and Automotive Engineering, Loughborough 1996. 